\title{
UNIVERSITY of York
}

This is a repository copy of Inverse Fourier transform technique of measuring averaged absorption cross section in the reverberation chamber and Monte Carlo study of its uncertainty.

White Rose Research Online URL for this paper:

https://eprints.whiterose.ac.uk/104691/

Version: Accepted Version

\section{Proceedings Paper:}

Zhang, Xiaotian, Robinson, Martin P orcid.org/0000-0003-1767-5541, Flintoft, lan D orcid.org/0000-0003-3153-8447 et al. (1 more author) (2016) Inverse Fourier transform technique of measuring averaged absorption cross section in the reverberation chamber and Monte Carlo study of its uncertainty. In: Proceedings of the 2016 International Symposium on Electromagnetic Compatibility - EMC EUROPE. EMC Europe 2016, 05-09 Sep 2016 IEEE , POL , pp. 263-267.

https://doi.org/10.1109/EMCEurope.2016.7739161

\section{Reuse}

Items deposited in White Rose Research Online are protected by copyright, with all rights reserved unless indicated otherwise. They may be downloaded and/or printed for private study, or other acts as permitted by national copyright laws. The publisher or other rights holders may allow further reproduction and re-use of the full text version. This is indicated by the licence information on the White Rose Research Online record for the item.

\section{Takedown}

If you consider content in White Rose Research Online to be in breach of UK law, please notify us by emailing eprints@whiterose.ac.uk including the URL of the record and the reason for the withdrawal request. 


\title{
Inverse Fourier Transform Technique of Measuring Averaged Absorption Cross Section in the Reverberation Chamber and Monte Carlo Study of its Uncertainty
}

\author{
Xiaotian Zhang, Martin P. Robinson, Ian D. Flintoft and John F. Dawson \\ Department of Electronics, University of York, Heslington, York YO10 5DD, UK
}

Published in the Proceedings of the International Symposium and Exhibition on Electromagnetic Compatibility, EMC Europe 2016, Wroclaw, Poland, pp. 263-267, 5-9 September 2016.

Accepted for publication: 21/04/2016

DOI: $\underline{\text { 10.1109/EMCEurope.2016.7739161 }}$

(C) 2016 IEEE. Personal use of this material is permitted. Permission from IEEE must be obtained for all other uses, in any current or future media, including reprinting/republishing this material for advertising or promotional purposes, creating new collective works, for resale or redistribution to servers or lists, or reuse of any copyrighted component of this work in other works. 


\title{
Inverse Fourier Transform Technique of Measuring Averaged Absorption Cross Section in the Reverberation Chamber and Monte Carlo Study of its Uncertainty
}

\author{
Xiaotian Zhang, Martin P. Robinson, Ian D. Flintoft and John F. Dawson \\ Department of Electronics \\ University of York \\ Email:xz1148@york.ac.uk, martin.robinson@york.ac.uk, ian.flintoft@york.ac.uk, john.dawson@york.ac.uk
}

\begin{abstract}
The averaged absorption cross section (ACS) of a lossy object characterises its ability to capture power from diffused electromagnetic waves. The averaged ACS is very important in many EMC research areas such as indoor wireless channel modelling and human safety exposure study. The measurement of averaged ACS in a reverberation chamber can be achieved by measuring the rate of power loss in the time domain, however this technique requires dense frequency sampling for taking the inverse Fourier transform, which is very time consuming. A new scenario which accelerates the measurement speed is presented in this paper. It combines the technique of non-linear curve fitting to the power delay profile, segmented frequency sweeping and continuous mode stirring. The scenario was validated by measuring the averaged ACS of a hollow plastic sphere filled with deionized water in an EMC reverberation chamber. Measurement results showed a good accordance with the simulations and the measurement uncertainty was studied numerically with the Monte Carlo method.

Keywords: averaged absorption cross section, reverberation chamber, segmented frequency sweeping, continuous mode stirring, non-linear curve fitting
\end{abstract}

\section{INTRODUCTION}

The absorption cross section (ACS) gives the amount of power that a lossy object can absorb from a incident planewave. Numerically, the value of ACS equals the ratio between the power dissipated in the object and power density of incident wave. Therefore it has a unit of $\mathrm{m}^{2}$. Physically, it can be studied in a way of studying an absorbing aperture [1].

In the diffused environments, such as multi-path indoor environments, it is very hard to set up a ideal single plane-wave configuration. So the ACS measured in diffused environments is the averaged ACS, which is the ACS averaged over different angles of incidence and polarisation of a plane-wave, according to Hill's theory [2]. The averaged ACS was found to be not only of a great importance to indoor channel modelling [3], but also very important in the study of non-ionizing radiation dosimetry [4]. Recently, it was found that ACS shows some correlation with morphology parameters of human body such as body mass index (BMI), average fat layer thickness [5], and body surface area [6], which showed its potential value in biomedical areas.
The measurement of averaged ACS can be performed in many indoor environments including aircraft cabins [7] and rooms [8]. Absorption effects also inform our understanding of reverberation chambers for EMC measurements of radiated emissions, radiated immunity and shielding [9], [10].

A reverberation chamber (RC) is a cavity containing a moving stirrer which helps in creating a stochastic field configuration inside. Therefore the lossy object inside a reverberation chamber is illuminated by electromagnetic waves coming from different directions when the stirrer moves from one position to another. The averaged ACS can be measured in two ways. Firstly, it can be done by measuring the average net power transfer function $G_{\text {chamber }}$ [1], [11], [12], [13], or it can be determined by measuring the change of the reverberation chamber time constant $\tau$ [14].

The former method comes from the idea of the power balance model in a reverberation chamber [1]. Thus by comparing the change of $G_{\text {chamber }}$ before and after the chamber is loaded, the power dissipated in the lossy object can be found [4]. This method is fast and easy to implement for broadband application, but the uncertainty of this method is dominated by the uncertainty of $S_{21}$ measurement, which is much larger than the measurement uncertainty of chamber time constant [15].

The second method is much more accurate than the first one, but it requires much denser frequency sampling, thus the measurement speed is lower. Further, due to the complicated data processing, it is not easy to apply for wideband applications, and it is very hard to give a full mathematical model for the measurement uncertainty. This paper will present some techniques of accelerating the measurement of reverberation chamber time constant and apply them to average ACS measurement. The techniques includes non-linear curve fitting, segmented frequency sweeping and continuous stirring. Experiments on measuring a spherical absorber were performed to test the effectiveness of the techniques, and finally the measurement uncertainty was evaluated with Monte Carlo method. 


\section{THEORY}

The reverberation chamber time constant $\tau$, is the time it takes for a reverberation chamber to lose its stored energy to a factor of $1 / e$ of the initial level after the input power is suddenly cut off [2]. So the shorter this time is, the lossier the inside of the reverberation chamber. The averaged ACS can be calculated by the following equations [1]:

$$
\begin{aligned}
<\sigma>=\frac{2 \pi V}{\lambda}\left(\frac{1}{<Q_{\mathrm{wo}}>}-\frac{1}{<Q_{\mathrm{no}}>}\right) \\
=\frac{V}{c}\left(\frac{1}{\left.<\tau_{\mathrm{wo}}\right\rangle}-\frac{1}{<\tau_{\mathrm{no}}>}\right)
\end{aligned}
$$

where $\lambda$ is the wavelength, $Q$ is the quality factor of the reverberation chamber, $V$ is the volume of the chamber and $c$ is the speed of light in the free space. The angle brackets $\langle\cdot\rangle$ mean the ensemble average over different stirrer positions, and the subscripts 'wo' and 'no' mean the value is measured when the chamber is loaded 'with object' or when 'no object' is loaded in the chamber repsectively. Equation (1) shows that the averaged ACS can be obtained directly by doing relative measurements of the chamber time constant.

However the wideband measurement of chamber time constant is not a straightforward task. First of all, the frequency sweeping rate should be low enough so that signal can die down before the measurement proceeds to the next frequency point [16]. Secondly, the scattering parameters in frequency domain should be densely sampled in order to give a long enough time domain response of power delay profile (PDP) from the slope of which the chamber time constant can be extracted [15]. And finally, the window function applied on the scattering parameters when taking the inverse Fourier Transform to determine the PDP should be carefully chosen, otherwise there will be an unexpected ringing 'tail' at the end of the PDP which can affect the evaluation of its slope. To tackle all these problems, the following techniques are introduced, which increase the measurement speed at the same time.

\section{A. Non-linear curve fitting}

The PDP can be calculated from $S_{21}$ using [17]

$$
\begin{aligned}
\operatorname{PDP}\left(t_{i}\right) & =<\left|h\left(t_{i}, n\right)\right|^{2}> \\
& =<\left|\operatorname{IFFT}\left[S_{21}\left(f_{k}, n\right) \cdot \operatorname{win}\left(f_{k}\right)\right]\right|>
\end{aligned}
$$

where $i$ is the index of the discrete samples in the time domain, $k$ is the index of the samples in the frequency domain and $h\left(t_{i}, n\right)$ is the impulse response of reverberation chamber at $n$ th stirrer position. "win" stands for the window function used in the inverse Fourier Transform. This mathematical form of the PDP shows the effect of the window function. According to the definition of the PDP, the slope of the PDP gives the chamber time constant.

For example, Fig. 1 and Fig. 2 show the PDP measured in the University of York reverberation chamber. The $S_{21}$ for calculating the PDP was filtered by a $5 \mathrm{MHz}$ raised cosine window with the roll-off factor set to $\beta=0.25$. These figures

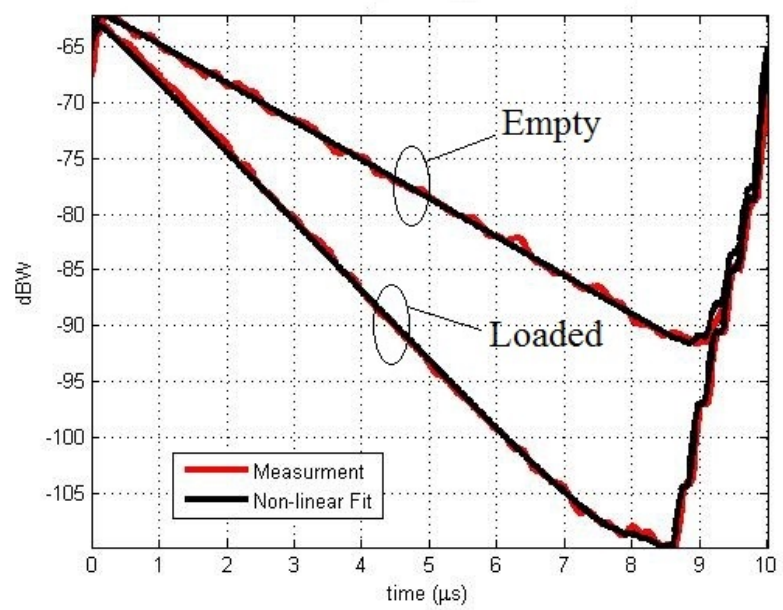

Fig. 1. PDP at $7 \mathrm{GHz}$ (5 $\mathrm{MHz}$ raised cosine window).

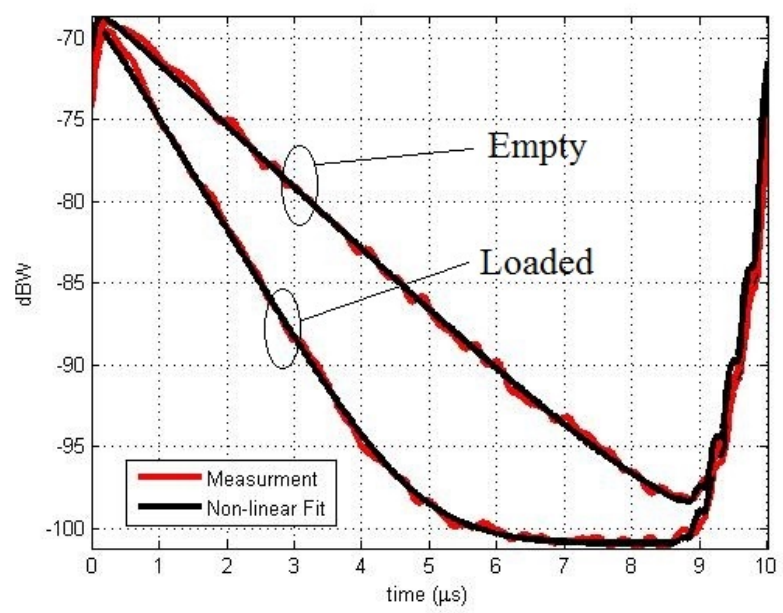

Fig. 2. $\mathrm{PDP}$ at $14 \mathrm{GHz}(5 \mathrm{MHz}$ raised cosine window).

show a change of the PDP slope when the chamber is loaded with a lossy object. It is possible to inspect the time domain response of the PDP at each frequency of interest, then simply pick out the linear part for the calculation of slope. However, it is very time consuming when there are many frequencies to be processed. Moreover, simply judging the boundary between the linear and non-linear part of PDP "by eye" is not reliable. For instance, in Fig. 2, the PDP of the loaded chamber just decays smoothly down into the noise floor at around -101 dBW.

To make the curve fitting more robust and more automatic the non-linear mathematical model of the PDP was introduced for accurate curve fitting [17]:

$$
\operatorname{PDP}_{\text {non-lin }}\left(t_{\mathrm{i}}\right)=\left[A \exp \left(-\frac{t_{\mathrm{i}}}{\tau}\right)+B\right] \widehat{N} \|\left|\operatorname{win}\left(t_{\mathrm{i}}\right)\right|^{2}
$$

where $A$ is the magnitude of the transmitted signal, $B$ is the magnitude of the noise floor, $\tau$ is chamber time constant. (N) denotes circulated convolution, and $N$ is both the length of 


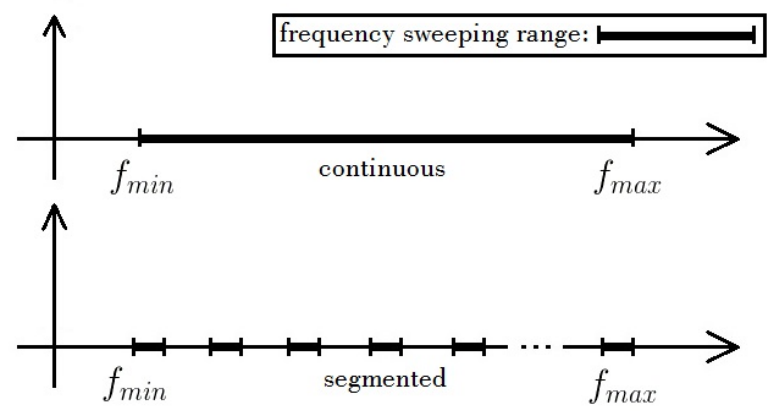

Fig. 3. Segmented frequency sweeping (below) compared to standard sweeping (above).

the convolution result and the length of window function. Then the Levenberg-Marquardt algorithm [18] was applied to search for the set of $A, B$, and $\tau$ which makes $\operatorname{PDP}_{\text {non-lin }}\left(t_{\mathrm{i}}\right)$ the best fit to the measurement result. The greatest advantage of nonlinear regression over linear regression is that it can cancel the distortion on the PDP due to the application of different types of window functions. Thus it makes the calculation of the chamber time constant more robust. Furthermore, the nonlinear model is good for fitting the whole range of PDP in the time domain, without the need for truncating the nonlinear part. So the time domain observation of the PDP at each frequency is no longer needed, thus making the calculation of chamber time constant more automatic.

\section{B. Segmented frequency sweeping}

Thanks to the robustness of the non-linear curve fitting, the shape and size of window function can be freely chosen. Therefore only a narrow band of $S_{21}$ is needed for calculating the chamber time constant at a specific frequency. The idea of segmented frequency sweeping is simple. Instead of sweeping over the whole band from minimum frequency to the maximum, it is much quicker to just sweep over a band around the frequencies of interest and neglect those which are not included in the IFFT calculation. The operation is illustrated in Fig. 3.

\section{Continuous stirring}

To improve the measurement speed even more, the continuous stirring technique is introduced. In the process of continuous stirring, the data collection is performed as the stirrer is constantly moving; however in stepped stirring, data is only collected when the stirrer stops at different positions. Because the change of electronic settings is much faster than the mechanical stirring, the continuous stirring and stepped stirring can be considered equivalent in some cases, and the related sampling techniques are stated in [19], [20].

In this paper the problem is approached in a empirical way. In the reverberation chamber measurement, it is always desired to get as many of the independent samples as possible. Therefore different stirring speeds were tested in order to
TABLE I

NUMBER OF INDEPENDENT SAMPLES IN REVERBERATION CHAMBER.

\begin{tabular}{|c|c|c|c|}
\hline$N_{\text {indep }}$ & Speed $=0.5625 \mathrm{deg} / \mathrm{s}$ & Speed $=1.125 \mathrm{deg} / \mathrm{s}$ & Stepped \\
\hline Freq $=1 \mathrm{GHz}$ & 247 & 185 & 200 \\
\hline Freq $=7 \mathrm{GHz}$ & 370 & 185 & 200 \\
\hline Freq $=13 \mathrm{GHz}$ & 370 & 185 & 200 \\
\hline Freq $=18 \mathrm{GHz}$ & 370 & 185 & 200 \\
\hline \hline$N_{\text {total }}$ & 740 & 370 & 200 \\
\hline \hline Measurement time $(\mathrm{sec})$ & 630 & 312 & 1461 \\
\hline
\end{tabular}

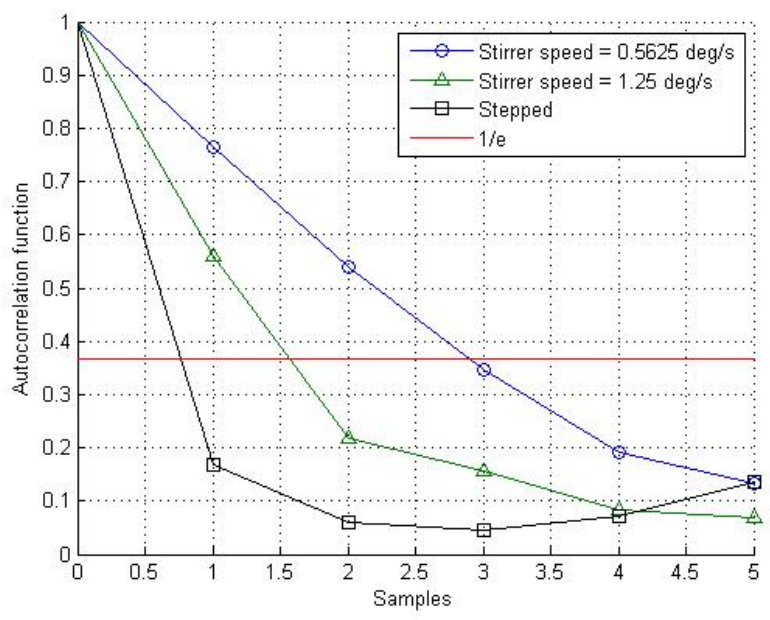

Fig. 4. The auto-correlation sequence of S21 over different stirrer postions when the chamber is empty.

see how many independent samples can be collected as the stirrer moves through 360 degrees, and at the same time, the measurement delay of the vector network analyser (VNA) was fixed at $65 \mu \mathrm{s}$ to give enough time for the whole system to settle. According to the standard IEC61000-1-24 [9], the number of independent samples can be calculated using

$$
N_{\text {indep }}=\frac{N_{\text {total }}}{N_{\text {corr }}},
$$

where $N_{\text {indep }}$ is the number of independent samples, $N_{\text {total }}$ is the number of all the samples collected in the measurement, $N_{\text {corr }}$ is the number of successive correlated samples, i.e. the number of consecutive samples for which the autocorrelation function is larger than $1 / e$. As an example, the autocorrelation functions of $S_{21}$ at $1 \mathrm{GHz}$ are plotted in Fig. 4. It shows that the $\mathrm{S} 21$ spectra have 3 successive correlated samples when the stirring speed is 0.5625 , while in other cases the number of successive correlated samples is less than 3. Equation 5 can be applied over all the frequencies. The number of independent samples at some frequencies are listed in Table I.

In real cases, the stirring speed can be chosen with reference to Table I, to make a trade off between measurement time and the number of independent samples collected.

\section{EXPERIMENT}

To validate the previously introduced techniques, a hollow plastic sphere filled with deionized water was tested in the University of York reverberation chamber. The size of the 


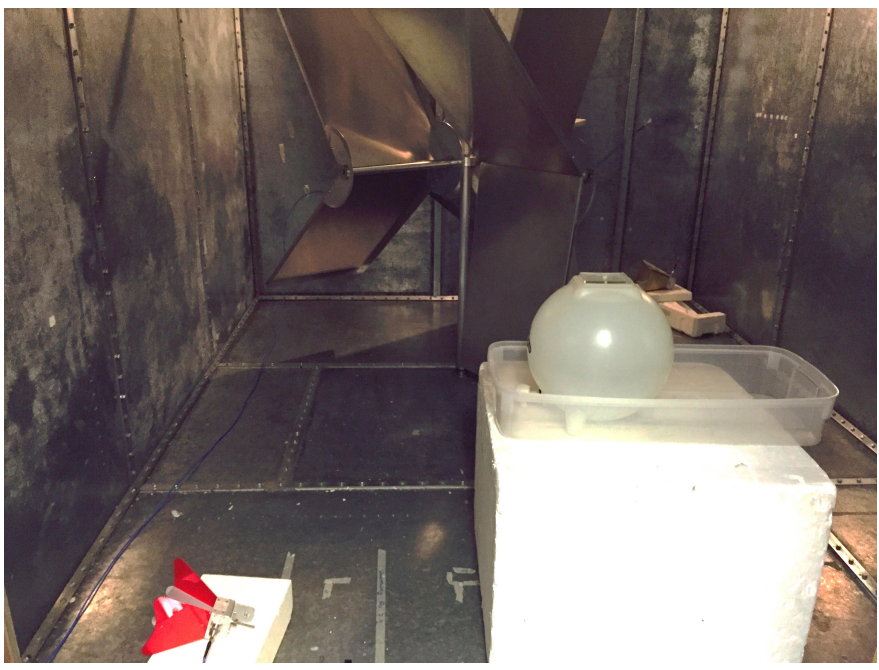

Fig. 5. Experiment setup in the University of York reverberation chamber, showing the paddle stirrer, broadband antennas and spherical absorber.

TABLE II

PARAMETERS OF THE MIE SERIES NUMERICAL MODEL.

\begin{tabular}{|c|c|c|}
\hline & Model No.1 & Model No.2 \\
\hline Radius (cm) & 19.10 & 19.50 \\
\hline Wall thickness (mm) & 3.9 & 3.9 \\
\hline Relative Permittivity of Sphere Wall & 2.3 (HDPE) & 2.1 (HDPE) \\
\hline
\end{tabular}

chamber is $4.70 \mathrm{~m} \times 3.00 \mathrm{~m} \times 2.37 \mathrm{~m}$. The VNA was connected to cross polarized horn antennas in the chamber to measure $S_{21}$. 171 frequencies from $1 \mathrm{GHz}$ to $18 \mathrm{GHz}$ were measured. The segments around the 171 frequencies were all 5 $\mathrm{MHz}$ wide. To achieve an overall measurement time of about 5 minutes, the stirrer was set to be continuously moving at a speed of $1.125 \mathrm{deg} / \mathrm{s}$. The experimental setup is shown in Fig. 5. The theoretical ACS of the sphere was calculated by the Mie code "SPlaC" [21] which can model a multi-layer sphere. Because the object under test is not perfectly spherical, two different sphere models were introduced as references. The parameters of the first model were obtained from calliper measurement of the radius and thickness of spherical shell; the second model's parameters are obtained by fitting the simulated ACS to the measurement data. Details of the two models are listed in Table II. Fig. 6 shows the measured ACS corresponds well with the simulations.

After the measurement, a preliminary study of the measurement uncertainty was done with a Monte Carlo method. Since the calculation of chamber time constant involves a lot of complex algorithms including IFFT, circulated convolution and Levenberg-Marquardt optimization, it is hard to derive the probability density function of the chamber time constant analytically, as can be done for the standard frequency domain approach [22]. However, taking advantage of the idea from 3 , the PDP at one stirrer position can be modelled using (6) $N_{\text {indep }}$ times as a random simulation of the measurement

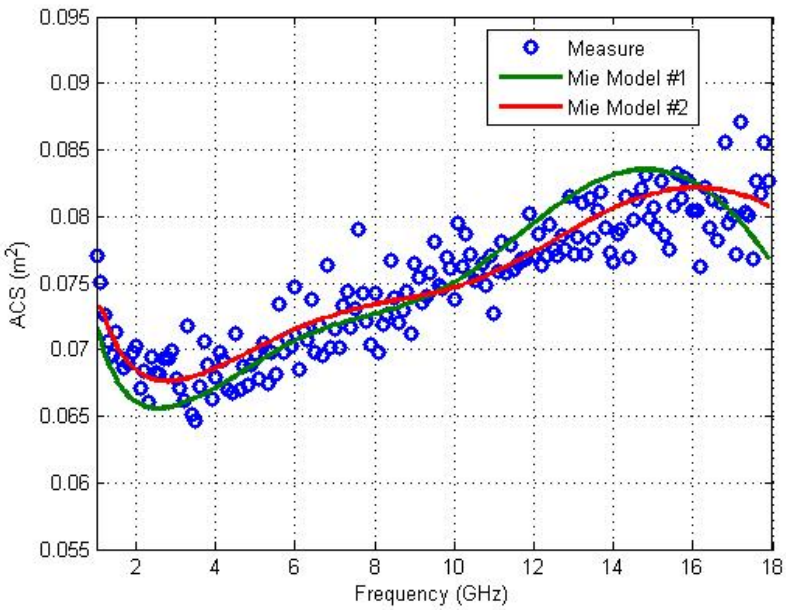

Fig. 6. Measured and modelled ACS of spherical test object.
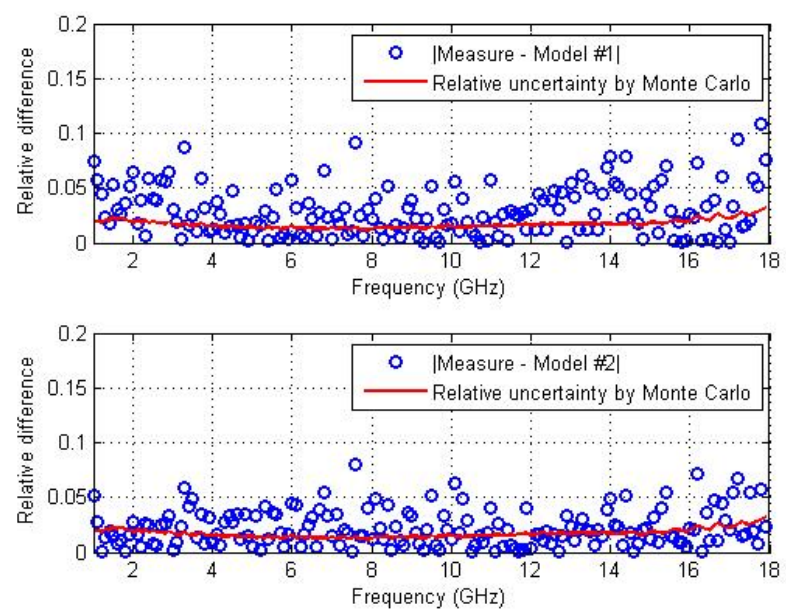

Fig. 7. The relative difference between measurement and simulation.

process:

$$
h\left(t_{i}, n\right)=\sqrt{A} \exp \left(-\frac{t_{\mathrm{i}}}{2 \tau}\right) N_{1}\left(t_{i}\right)+\sqrt{B} N_{2}\left(t_{i}\right),
$$

where $N_{1}\left(t_{i}\right)$ and $N_{2}\left(t_{i}\right)$ are two independent complex Gaussian random processes with zero mean and a variance of one. The PDP sets are generated and fitted 500 times to get a group of ACS results. Finally the relative uncertainty of measurement was obtained by normalizing the standard deviation of the generated ACS with the simulation values. The relative uncertainties are plotted as red lines in Fig. 7.

Even though the relative differences are all well below $10 \%$ from $1 \mathrm{GHz}$ to $18 \mathrm{GHz}$, there are still a lot of samples above the boundary of relative uncertainty given by the Monte Carlo method. It is important to point out that the Monte Carlo method just gives the uncertainty coming from the statistical data processing. However in real cases, the measurement uncertainty would be larger than the Monte Carlo predictions 
due to other sources of error, such as the fluctuation of temperature, impurities of the materials under test, imperfect shapes of the object under test, and so on.

\section{CONCLUSION}

The use of combined non-linear curve fitting, segmented frequency sweeping and continuous stirring techniques applied to averaged ACS measurement in a reverberation chamber were validated by measuring a hollow plastic sphere filled with deionized water in the University of York reverberation chamber. The measurement time was less than $5 \mathrm{~min}$. The measurement results were compared with two numerical models. Both comparisons showed the relative differences were well below 10\%, which showed the high efficiency and accuracy of the new techniques.

A preliminary study on the measurement uncertainty was also made using the Monte Carlo method. The Monte Carlo study estimates the statistical uncertainty as below 5\% from 1 $\mathrm{GHz}-18 \mathrm{GHz}$; however, in real cases, the relative uncertainty could be larger than this value, due to other non ideal factors in the experimental environment. The results of this work give us confidence that rapid, accurate measurements of ACS are feasible, making this a promising techniques for EMC and human exposure studies.

\section{REFERENCES}

[1] D. Hill, M. T. Ma, A. R. Ondrejka, B. F. Riddle, M. L. Crawford, R. T Johnk et al., "Aperture excitation of electrically large, lossy cavities," Electromagnetic Compatibility, IEEE Transactions on, vol. 36, no. 3, pp. 169-178, 1994

[2] D. A. Hill, Electromagnetic fields in cavities: deterministic and statistical theories. John Wiley \& Sons, 2009, vol. 35.

[3] C. L. Holloway, D. A. Hill, J. M. Ladbury, P. F. Wilson, G. Koepke, and J. Coder, "On the use of reverberation chambers to simulate a Rician radio environment for the testing of wireless devices," Antennas and Propagation, IEEE Transactions on, vol. 54, no. 11, pp. 3167-3177, 2006.

[4] G. C. R. Melia, M. P. Robinson, I. D. Flintoft, A. C. Marvin, and J. F. Dawson, "Broadband measurement of absorption cross section of the human body in a reverberation chamber," Electromagnetic Compatibility, IEEE Transactions on, vol. 55, no. 6, pp. 1043-1050, 2013.

[5] I. D. Flintoft, M. P. Robinson, G. C. R. Melia, A. C. Marvin, and J. F. Dawson, "Average absorption cross-section of the human body measured at $1-12 \mathrm{GHz}$ in a reverberant chamber: results of a human volunteer study," Physics in Medicine and Biology, vol. 59, no. 13, p. 3297, 2014

[6] A. Hirata, Y. Nagaya, F. Osamu, T. Nagaoka, and S. Watanabe, "Correlation between absorption cross section and body surface area of human for far-field exposure at $\mathrm{GHz}$ bands," in Electromagnetic Compatibility, 2007. EMC 2007. IEEE International Symposium on, July 2007, pp. $1-4$.

[7] J. B. Andersen, K. L. Chee, M. Jacob, G. F. Pedersen, and T. Kurner, "Reverberation and absorption in an aircraft cabin with the impact of passengers," Antennas and Propagation, IEEE Transactions on, vol. 60, no. 5, pp. 2472-2480, May 2012.

[8] A. Bamba, W. Joseph, J. B. Andersen, E. Tanghe, G. Vermeeren, D. Plets, J. O. Nielsen, and L. Martens, "Experimental assessment of specific absorption rate using room electromagnetics," Electromagnetic Compatibility, IEEE Transactions on, vol. 54, no. 4, pp. 747-757, Aug 2012.

[9] Electromagnetic compatibility (EMC) - Part 4-21: Testing and measurement techniques - Reverberation chamber test methods, International Electrotechnical Commission Std. IEC 61 000-4-21:2011, Rev. 2.0, 27 January 2011
[10] I. D. Flintoft, S. L. Parker, S. J. Bale, A. C. Marvin, J. F. Dawson, and M. P. Robinson, "Measured average absorption cross-sections of printed circuit boards from 2 to $20 \mathrm{GHz}$," Electromagnetic Compatibility, IEEE Transactions on, 2016, in press.

[11] U. Carlberg, P.-S. Kildal, A. Wolfgang, O. Sotoudeh, and C. Orlenius, "Calculated and measured absorption cross sections of lossy objects in reverberation chamber," Electromagnetic Compatibility, IEEE Transactions on, vol. 46, no. 2, pp. 146-154, May 2004.

[12] A. Gifuni, "On the measurement of the absorption cross section and material reflectivity in a reverberation chamber," Electromagnetic Compatibility, IEEE Transactions on, vol. 51, no. 4, pp. 1047-1050, Nov 2009.

[13] I. D. Flintoft, G. C. R. Melia, M. P. Robinson, J. F. Dawson, and A. C. Marvin, "Rapid and accurate broadband absorption crosssection measurement of human bodies in a reverberation chamber," Measurement Science and Technology, vol. 26, no. 6, p. 065701, 2015. [Online]. Available: http://stacks.iop.org/0957-0233/26/i=6/a=065701

[14] A. Bamba, D. P. Gaillot, E. Tanghe, G. Vermeeren, W. Joseph, M. Lienard, and L. Martens, "Assessing whole-body absorption cross section for diffuse exposure from reverberation chamber measurements," Electromagnetic Compatibility, IEEE Transactions on, vol. 57, no. 1, pp. 27-34, Feb 2015

[15] C. L. Holloway, H. A. Shah, R. J. Pirkl, W. F. Young, D. A. Hill, and J. Ladbury, "Reverberation chamber techniques for determining the radiation and total efficiency of antennas," Antennas and Propagation, IEEE Transactions on, vol. 60, no. 4, pp. 1758-1770, 2012

[16] L. R. Arnaut, "Maximum rate of frequency scanning for distortionless signal generation in electromagnetic reverberation chambers," Electromagnetic Compatibility, IEEE Transactions on, vol. 50, no. 4, pp. 787793, Nov 2008

[17] X. Zhang, M. P. Robinson, and I. D. Flintoft, "On measurement of reverberation chamber time constant and related curve fitting techniques," in Electromagnetic Compatibility (EMC), 2015 IEEE International Symposium on, Aug 2015, pp. 406-411.

[18] D. W. Marquardt, "An algorithm for least-squares estimation of nonlinear parameters," Journal of the Society for Industrial and Applied Mathematics, vol. 11, no. 2, pp. 431-441, 1963.

[19] V. Rajamani, C. F. Bunting, and J. C. West, "Stirred-mode operation of reverberation chambers for EMC testing," Instrumentation and Measurement, IEEE Transactions on, vol. 61, no. 10, pp. 2759-2764, Oct 2012.

[20] — "Sensitivity analysis of a reverberation chamber with respect to tuner speeds," in Electromagnetic Compatibility, 2007. EMC 2007. IEEE International Symposium on. IEEE, 2007, pp. 1-6.

[21] E. Le $\mathrm{Ru}$ and P. Etchegoin, SPlaC package $v 1.0$ guide and supplementary information, 1st ed., Raman Laboratory, Victoria University of Wellington, New Zealand, 3 December 2008. [Online]. Available: http://www.victoria.ac.nz/scps/research/researchgroups/raman-lab/numerical-tools/sers-and-plasmonics-codes

[22] A. Gifuni, G. Ferrara, A. Sorrentino, and M. Migliaccio, "Analysis of the measurement uncertainty of the absorption cross section in a reverberation chamber," Electromagnetic Compatibility, IEEE Transactions on, vol. 57, no. 5, pp. 1262-1265, Oct 2015. 